\title{
Nonlinear Dynamic Modeling and Optimal Motion Analysis of Two-Link Manipulators
}

\author{
M. Talezadeh*, M. Ghazal*, M. Taheri*, M. Nazemi-Zade* \\ * Department of Mechanics, Damavand Branch, Islamic Azad University, Damavand, Iran
}

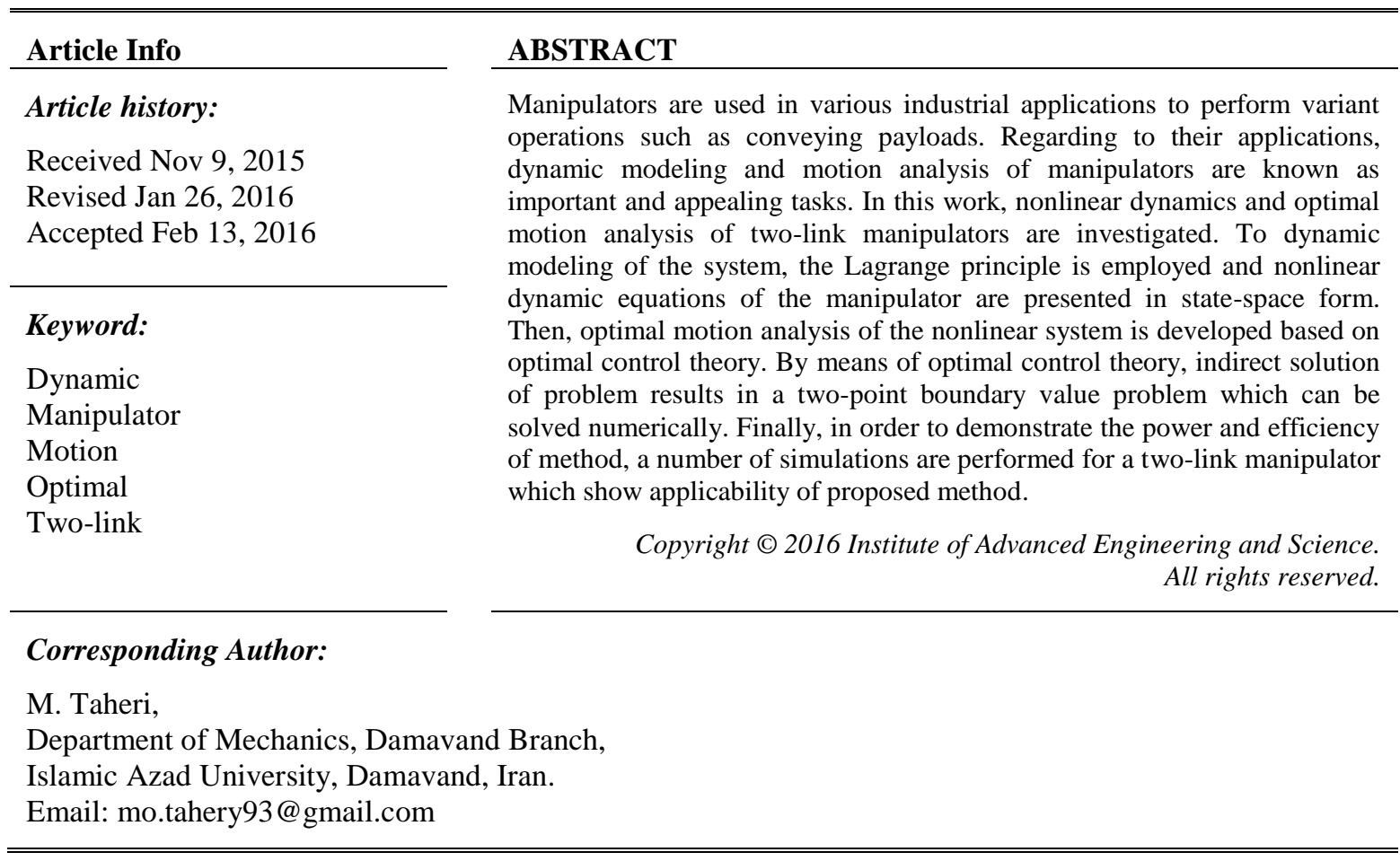

\section{INTRODUCTION}

Fixed and mobile Manipulators are used in many applications and perform several tasks such as conveying payloads, rehabilitation tasks and etc [1-6]. In fact, due to their advantage of high speed, accuracy and repeatability, robot manipulators have become major component of industrial applications and even now a days they become part of routine life. Regarding to their vast applications, dynamic modeling and motion analysis of such systems have attracted a great deal of interests by many robotic researchers. It is well known that robot manipulators are highly nonlinear, dynamically coupled and time-varying systems and their dynamic analysis is a complex and challenging issue. Luh [7] studied industrial manipulators and developed some conventional method to control dynamic motion of the manipulators. Song et al. [8] investigated dynamic motion of robotic manipulators and proposed a computed torque controller to handle requirement of precise dynamical models of robotic manipulators. Piltan et al. [9] presented dynamic motion of robotic manipulators and developed a nonlinear control strategy to motion control of highly nonlinear dynamic robot manipulator in presence of uncertainties. Rahimi et al. [10] studied dynamic analysis of elastic manipulators. They investigated trajectory optimization of such robot using optimal control theory. Moreover, they [11] proposed finite element method to model dynamics of elastic manipulators.

In this work, nonlinear dynamics and optimal motion planning of two-link manipulators are investigated. To dynamic modeling of the system, the Lagrange principle is employed and nonlinear dynamic equations of the manipulator are presented in state-space form. Then, optimal motion analysis of the nonlinear system is developed based on optimal control theory. By means of optimal control theory, indirect solution of results in a two-point boundary value problem which can be solved numerically. Finally, in order to demonstrate the power and efficiency of method, a number of simulations are performed for a two-link manipulator which show applicability of proposed method. 


\section{DYNAMIC MODEL OF MANIPULATOR} end-effetor

As it is seen in Figure 1, a two-link manipulator is presented. The manipulator has two links and an

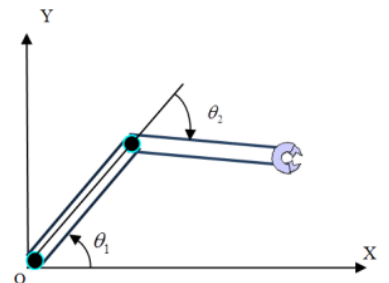

Figure 1. The two-link manipulator

The parameters of the two-link manipulator are defined as: $\theta_{1}$ is the angular displacement of the first link of the manipulator, $\theta_{2}$ is the angular displacement of the second link, $\dot{\theta}_{1}$ is the angular velocity of the first link, $\dot{\theta}_{2}$ is the angular velocity of the second link, $L_{1}$ is the length of the first link of the manipulator, $L_{2}$ is the length of the seond link of the manipulator, $m_{1}$ is the mass of the first link of the manipulator, $m_{2}$ is the mass of the second link of the manipulator, $m_{p}$ is the mass of the payload and endeffector of the manipulator, $I_{1}$ is the moment of inertia of the first link and $I_{2}$ is the moment of inertia of the second link.

To derive nonlinear dynamic equations of the manipulator, the kinetic energy of the manipulator $\mathrm{T}$ and the potential energy $\mathrm{U}$ is stated as:

$$
\begin{gathered}
T=\frac{1}{2} m_{1}\left(\dot{x}_{c 1}^{2}+\dot{y}_{c 1}^{2}\right)+\frac{1}{2} I_{1} \dot{\theta}_{1}^{2}+\frac{1}{2} m_{2}\left(\dot{x}_{c 2}^{2}+\dot{y}_{c 2}^{2}\right)+\frac{1}{2} I_{2} \dot{\theta}_{2}^{2}+\frac{1}{2} m_{p}\left(\dot{x}_{f}^{2}+\dot{y}_{f}^{2}\right) \\
U=m_{1} g \frac{L_{1}}{2} \sin \theta_{1}+m_{2} g\left(L_{1} \sin \theta_{1}+\frac{L_{2}}{2} \sin \left(\theta_{1}+\theta_{2}\right)\right)+m_{p} g\left(L_{1} \sin \theta_{1}+L_{2} \sin \left(\theta_{1}+\theta_{2}\right)\right)
\end{gathered}
$$

where the velocities of each link and end-effector are given as:

$$
\begin{aligned}
& \dot{x}_{c 1}=-\frac{L_{1}}{2} \dot{\theta}_{1} \sin \theta_{1} \\
& \dot{y}_{c 1}=\frac{L_{1}}{2} \dot{\theta}_{1} \cos \theta_{1} \\
& \dot{x}_{c 2}=-L_{1} \dot{\theta}_{1} \sin \theta_{1}-\frac{L_{2}}{2}\left(\dot{\theta}_{1}+\dot{\theta}_{2}\right) \sin \left(\theta_{1}+\theta_{2}\right) \\
& \dot{y}_{c 2}=L_{1} \dot{\theta}_{1} \cos \theta_{1}+\frac{L_{2}}{2}\left(\dot{\theta}_{1}+\dot{\theta}_{2}\right) \cos \left(\theta_{1}+\theta_{2}\right) \\
& \dot{x}_{p}=-L_{1} \dot{\theta}_{1} \sin \theta_{1}-L_{2}\left(\dot{\theta}_{1}+\dot{\theta}_{2}\right) \sin \left(\theta_{1}+\theta_{2}\right)
\end{aligned}
$$


$\dot{y}_{p}=L_{1} \dot{\theta}_{1} \cos \theta_{1}+L_{2}\left(\dot{\theta}_{1}+\dot{\theta}_{2}\right) \cos \left(\theta_{1}+\theta_{2}\right)$

As the dynamic equations of the robot are developed by Lagrange principle, the Lagrangian function $(L=T-U)$ is calculated and substituted in the Lagrangian equation:

$$
\frac{d}{d t}\left(\frac{\partial L}{\partial \dot{q}_{i}}\right)-\frac{\partial L}{\partial q_{i}}=Q_{i}
$$

where is the generalized force related to the generalize coordinate. Now, using Lagrangian equation the nonlinear dynamic equations of the system can be obtained in the compact form as:

$$
M \ddot{\vec{q}}+\vec{V}(\vec{q}, \dot{\vec{q}})=B \vec{\tau}
$$

In which $\vec{\tau} \in R^{n}$ is torque vector exerted to the joints, $M(\vec{q}) \in R^{n \times n}$ is the inertia matrix, $B$ is constant input matrix, $\vec{V}(\vec{q}, \dot{\vec{q}}) \in R^{n}$ is a vector which presents coriolis and gravitational forces. The above matrices are given as:

$$
\begin{gathered}
M=\left[\begin{array}{cc}
\frac{1}{3} m_{1} L_{1}^{2}+m_{2} L_{1}^{2}+\frac{1}{3} m_{2} L_{2}^{2}+m_{p}\left(L_{1}^{2}+L_{2}^{2}\right)+m_{2} L_{1} L_{2} \cos \theta_{2}+2 m_{p} L_{1} L_{2} \cos \theta_{2} & \frac{1}{3} m_{2} L_{2}^{2}+m_{p} L_{2}^{2}+\frac{1}{2} m_{p} L_{2}^{2} m_{2} L_{1} L_{2} \cos \theta_{2}+m_{p} L_{1} L_{2} \cos \theta_{2} \\
\frac{1}{3} m_{2} L_{2}^{2}+m_{p} L_{2}^{2}+\frac{1}{2} m_{p} L_{2}^{2} m_{2} L_{1} L_{2} \cos \theta_{2}+m_{p} L_{1} L_{2} \cos \theta_{2} & \frac{1}{3}\left(m_{2}+3 m_{p}\right) L_{2}^{2}
\end{array}\right] \\
\vec{V}(\vec{q}, \dot{\vec{q}})=\left[\begin{array}{c}
-\frac{1}{2}\left(m_{2}+2 m_{p}\right) L_{1} L_{2} \dot{\theta}_{2}\left(2 \dot{\theta}_{1}+\dot{\theta}_{2}\right) \sin \theta_{2} \\
\frac{1}{2}\left(m_{2}+2 m_{p}\right) L_{1} L_{2} \dot{\theta}_{1}^{2} \sin \theta_{2}
\end{array}\right] \\
B=\left[\begin{array}{cc}
1 & 0 \\
0 & 1
\end{array}\right]
\end{gathered}
$$

Furthermore, the nonlinear equations of the robot in state-space form are given as:

$$
\dot{X}=\left[\begin{array}{c}
x_{3} \\
x_{4} \\
M^{-1}(B \vec{\tau}-\vec{V})
\end{array}\right]
$$

where the state vector is $\dot{X}=\left[\begin{array}{llll}x_{1} & x_{2} & x_{3} & x_{4}\end{array}\right]^{T}=\left[\begin{array}{llll}\theta_{1} & \theta_{2} & \dot{\theta}_{1} & \dot{\theta}_{2}\end{array}\right]^{T}$ and the torque vector is related to torques exerted to the first and second links

\section{OPTIMAL MOTION ANALYSIS}

The optimal control theory is widely used many robotic applications [12-14]. In this section, optimal motion of the two-link manipulator is analyzed. To do this, optimal ontrol theory is employed. The dynamic equations of mobile robot in state space form is presumed as constraints of optimal control problem and it is aimed to determine optimal state vector $\mathrm{X}^{*}$ and optimal control vector $\mathrm{u}^{*}$ which following objective function can be minimized [11]: 


$$
J(X, u)=\int_{t_{0}}^{t_{f}} L(X(t), u(t), t) d t
$$

The indirect solution of optimal control problem is presented which begins from forming the Hamiltonian function $H=L+\psi^{T} \dot{X}$ where $\psi$ is denoted as co-state vector. Then necessary conditions for optimal motion are obtained as the following equation which is a two point boundary value problem:

$$
\begin{aligned}
& \dot{X}^{*}(t)=\frac{\partial H}{\partial \Psi}\left(X^{*}(t), u^{*}(t), \Psi^{*}(t), t\right) \\
& \dot{\Psi}^{*}(t)=-\frac{\partial H}{\partial X}\left(X^{*}(t), u^{*}(t), \Psi^{*}(t), t\right) \\
& 0=\frac{\partial H}{\partial u}\left(X^{*}(t), u^{*}(t), \Psi^{*}(t), t\right)
\end{aligned}
$$

But determining the appropriate cost function is an important task in optimal control formulation and must be considered thoroughly. For optimal motion planning of mobile robot the cost function is assumed as a minimum energy function which includes speed and torque of actuators. Therefore, the cost function can be rewritten as:

$$
J(X, u)=\int_{t_{0}}^{t_{f}} L(X(t), u(t), t) d t=\int_{t_{0}}^{t_{f}}\left(\frac{1}{2}\|X\|_{W}^{2}+\frac{1}{2}\|u\|_{R}^{2}\right) d t
$$

where $\|X\|_{W}^{2}$ is the generalized squared norm of state vector with respect to state weighting matrix $W$ and $\|u\|_{R}^{2}$ is generalized squared norm of control vector with respect to control weighting matrix $R$

\section{SIMULATION RESULTS}

In this section, dynamic motion of the two-link manipulator is simulated. The values of the parameters are given as: $m_{1}=2 \mathrm{~kg}, m_{2}=2 \mathrm{~kg}, m_{p}=1 \mathrm{~kg}, L_{1}=1 \mathrm{~m}$ and $L_{2}=1 \mathrm{~m}$.

To simulate the optimal motion of the manipulator, it is assumed that the robot moves from initial position $\left(\theta_{1}=0 \mathrm{rad}, \theta_{2}=\pi / 12 \mathrm{rad}, \dot{\theta}_{1}=0 \mathrm{rad} / \mathrm{s}, \dot{\theta}_{2}=0 \mathrm{rad} / \mathrm{s}\right)$ to final position $\left(\theta_{1}=3 \pi / 4 \mathrm{rad}\right.$, $\theta_{2}=\pi / 2 \mathrm{rad}, \dot{\theta}_{1}=0 \mathrm{rad} / \mathrm{s}, \dot{\theta}_{2}=0 \mathrm{rad} / \mathrm{s}$ ) during time of $t_{f}=1.2 \mathrm{~s}$. The path of the robot is shown in figure (2):

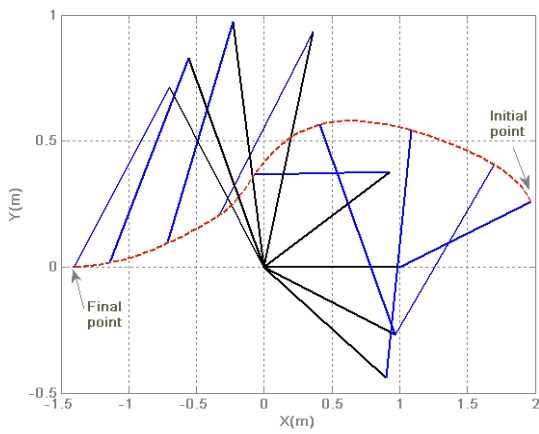


Figure 2. path of the manipulator

As it is seen in Figure 2, dynamic motion of the manipulator is simulated regarding to derived nonlinear equations of the robot. Moreover, the angular displacements of links of the robot are shown in figures (3) and (4):

As it is seen in above figures, the angular displacements of the robot are smooth. Furthermore, the velocities of the right and left fixed wheels of the robot are presented as:

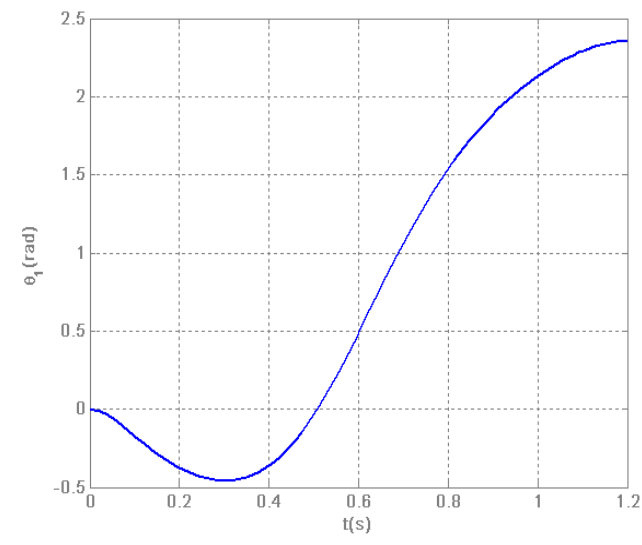

Figure 3. Angular displacement of first link

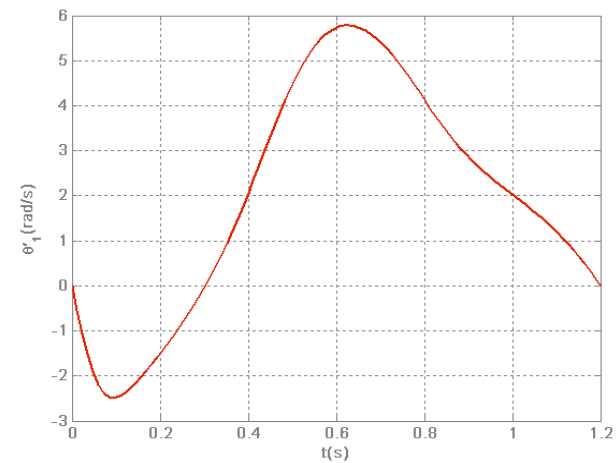

Figure 5. speed of the first link of the robot

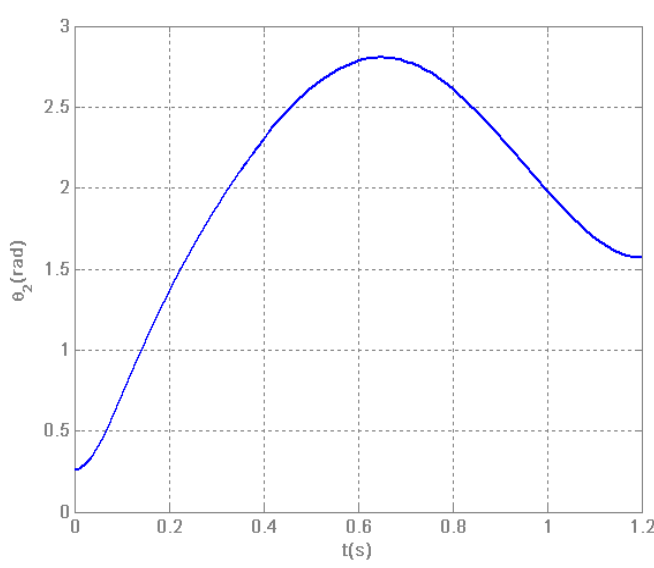

Figure 4. Angular displacement of second link

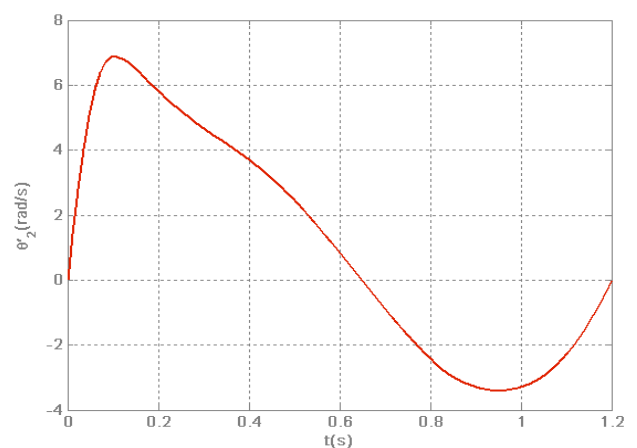

Figure 6. speed of the second link

As it is seen in simulation study, optimal dynamic motion of the manipulator is simulated based on derived equations of the system.

\section{CONCLUSION}

In this article, nonlinear dynamics and optimal motion of two-link manipulators have been investigated. To dynamic modeling of the system, the Lagrange principle has been employed and nonlinear dynamic equations of the manipulator have been presented in state-space form. Then, optimal motion analysis of the nonlinear system has been developed based on optimal control theory. By means of optimal control theory, indirect solution of problem has been resulted in a two-point boundary value problem which can be solved numerically. Finally, in order to demonstrate the power and efficiency of method, a number of simulations have been performed for a two-link manipulator which show applicability of the proposed method.

\section{REFERENCES}

[1] Piltan, F., Haghighi, S. T., Sulaiman, N., Nazari, I., \& Siamak, S. (2011). Artificial Control of PUMA Robot Manipulator: A-Review of Fuzzy Inference Engine And Application to Classical Controller. International Journal of Robotics and Automation, 2(5), 401-425. 
[2] Tondu, B., Ippolito, S., Guiochet, J., \& Daidie, A. (2005). A seven-degrees-of-freedom robot-arm driven by pneumatic artificial muscles for humanoid robots. The International Journal of Robotics Research, 24(4), 257-274

[3] Rahimi, H. N., \& Nazemizadeh, M. (2014). Dynamic analysis and intelligent control techniques for flexible manipulators: a review. Advanced Robotics,28(2), 63-76.

[4] Korayem, M. H., Nazemizadeh, M., \& Rahimi, H. N. (2013). Trajectory optimization of nonholonomic mobile manipulators departing to a moving target amidst moving obstacles. Acta Mechanica, 224(5), 995-1008

[5] Korayem, M. H., Rahimi, H. N., Nikoobin, A., \& Nazemizadeh, M. (2013). Maximum Allowable Dynamic Payload for Flexible Mobile Robotic Manipulators. Latin American Applied Research, 43(1), 29-35

[6] Korayem, M. H., Nazemizadeh, M., \& Azimirad, V. (2011). Optimal trajectory planning of wheeled mobile manipulators in cluttered environments using potential functions. Scientia Iranica, 18(5), 1138-1147

[7] Luh, J.Y.S., "Conventional controller design for industrial robots - A tutorial," Systems, Man and Cybernetics, IEEE Transactions on, vol.SMC-13, no.3, pp.298,316, May-June 1983

[8] Song, Z., Yi, J., Zhao, D., \& Li, X. (2005). A computed torque controller for uncertain robotic manipulator systems: Fuzzy approach. Fuzzy Sets and Systems, 154(2), 208-226

[9] Piltan, F., Mehrara, S., Bayat, R., \& Rahmdel, S. (2012). Design New Control Methodology of Industrial Robot Manipulator: Sliding Mode Baseline Methodology

[10] Korayem, M. H., \& Nohooji, H. R. (2008). Trajectory optimization of flexible mobile manipulators using openloop optimal control method. In Intelligent Robotics and Applications (pp. 54-63). Springer Berlin Heidelberg

[11] Korayem, M. H., Haghpanahi, M., Rahimi, H. N., \& Nikoobin, A. (2009). Finite element method and optimal control theory for path planning of elastic manipulators. In New Advances in Intelligent Decision Technologies (pp. 117-126). Springer Berlin Heidelberg

[12] Korayem, M. H., Nazemizadeh, M., \& Nohooji, H. R. (2012). Smooth jerk-bounded optimal path planning of tricycle wheeled mobile manipulators in the presence of environmental obstacles. Int J Adv Robotic Sy, 9(105)

[13] Korayem, M. H., Nazemizadeh, M., \& Rahimi, H. N. (2014). Dynamic optimal payload path planning of mobile manipulators among moving obstacles.Advanced Robotics, 28(20), 1389-1402

[14] Korayem, M. H., Nazemizadeh, M., \& Nohooji, H. R. (2014). Optimal point-to-point motion planning of nonholonomic mobile robots in the presence of multiple obstacles. Journal of the Brazilian Society of Mechanical Sciences and Engineering, 36(1), 221-232 